$63^{\text {ème }}$ Congrès de la SFCO, 02020 (2015)

DOI:10.1051/sfco/20156302020

(C) Owned by the authors, published by EDP Sciences, 2015

\title{
COMMUNICATION
}

\section{Forme atypique de la tuberculose orale révélatrice d'une tuberculose pulmonaire : à propos d'un cas clinique}

\author{
Farouk M, Haitami S, Ben Yahya I \\ Faculté de médecine dentaire de Casablanca, Rue Abou Al Alaa Zahar 21100, BP. 9157 Mers Sultan, \\ Casablanca, Maroc
}

La tuberculose est une maladie infectieuse causée par le bacille Mycobacteriumtuberculosis. Elle est principalement transmise par voie respiratoire. C'est un problème de santé mondiale, avec huit millions de personnes nouvellement infectées chaque année et trois millions de décès liés aux complications de la maladie (Renu 2012).

La tuberculose peut être pulmonaire, la forme la plus commune de la maladie, ou extra pulmonaire au niveau des ganglions lymphatiques, des méninges, des reins, des os, de la peau et dans le cavité orale (Shamimul 2011).

La tuberculose de la cavité orale est une entité rare, qui peut être primaire ou secondaire (Rasmané 2013).

Les lésions primaires sont extrêmement rares et apparaissent généralement chez les jeunes adultes, en rapport avec une contamination orale directe par le bacille. Cliniquement la lésion primaire est souvent associée à une adénopathie cervicale indolore accompagnant un chancre sous forme d'une petite ulcération à fond rouge ou fibrineux jaunâtre. Celui-ci cicatrisera en quelques semaines mais l'adénopathie persistera et évoluera vers une fibrose (Arnaud 2013).

La localisation secondaire est fréquente ( $0,005 \%$ à $1,5 \%$ des cas), elle est due à l'infection des tissus par dissémination hématogène, lymphatique, ou par auto inoculation par crachats infectés. La forme secondaire miliaire se présente sous forme de nappes érythémateuses et granuleuses jaunâtres qui s'ulcèrent et se couvrent d'exsudat fibrineux, alors que la forme de lupus se présente sous forme de plaques rouges violacées dont l'évolution est lente et très mutilante, pouvant parfois se compliquer par l'apparition d'un carcinome épidermoïde (Arnaud 2013).

Cependant en présence d'une forme de tuberculose buccale et même en absence de symptômes généraux, la recherche d'une localisation pulmonaire est obligatoire (Chang 2014).

Des forme atypiques de tuberculose buccale ont été décrites dans la littérature ce qui nécessite une vigilance du médecin dentiste dans le diagnostic et le dépistage de cette maladie.

Ce travail rapporte le cas d’une femme de 45 ans édentée totale, adressée par le service de prothèse adjointe pour chirurgie pré-prothétique.

L'anamnèse n'a signalé aucun élément particulier.

L'examen clinique a montré la présence au niveau des sommets des deux crêtes, maxillaire et mandibulaires de multiples hypertrophies avec une muqueuse de recouvrement d'aspect normal.

Une biopsie à été faite avant la réalisation de la chirurgie par bistouri électrique.

L’examen anatomopathologique a révélé une tuberculose.

This is an Open Access article distributed under the terms of the Creative Commons Attribution License 4.0, which permits unrestricted use, distribution, and reproduction in any medium, provided the original work is properly cited. 
Un test d'IDR a été demandé dont le résultat était de $17 \mathrm{~mm}$.

La patiente a été adressée au service de pneumologie qui a confirmé l'atteinte pulmonaire. Un traitement antituberculeux a été instauré.

Nom et adresse du conférencier

Mohammed FAROUK

Faculté de médecine dentaire de Casablanca

Rue Abou Al Alaa Zahar 21100, BP. 9157 Mers Sultan Casablanca

Casablanca (Maroc)

mohammed.dentiste@gmail.com 